\title{
Improved Parallel Lane Detection Using Modified Additive Hough Transform
}

\author{
Amandeep Katru \\ Guru Nanak Dev University, Amritsar, 143001, India \\ Enail: amandeepkatru@gmail.com \\ Anil Kumar \\ Guru Nanak Dev University, Amritsar, 143001, India \\ Enail: anil.dcse@gndu.ac.in
}

\begin{abstract}
Lane detection has recognition in real time vehicular ad-hoc system. That study work concentrate on giving greater efficiency in lane detection by utilizing the additive Hough transform to identify the curve lanes and convert into data parallelism in order to improve the speed of the proposed technique by using fork and join process. To accomplish performance evaluation various metrics is likely to be considered. The performance of lane detection algorithms is generally evaluated in terms of algorithm results and parallel results. Algorithm results is evaluated in terms of accuracy, error rate, execution time, overhead and parallel results is evaluated in terms of speed, efficiency etc. To complete performance comparison the result of proposed algorithm is going to be compared with existing lane detection algorithms. Intelligent transportation systems are available these days for increasing the safety of the vehicles and reduce incident ratio. A new technique which uses modified additive hough transform is used to reduce the limitations of existing technique. The proposed algorithm has been designed and implemented in MATLAB.
\end{abstract}

Index Terms - Lane detection, Modified additive Hough transfom, parallelisms, dynamic thresholding.

\section{INTRODUCTION}

Many people's die every day because of road accidents .Lane detection is used to reduce the road accident and also helps to improve the conditions of traffics. Lane detection helps the drivers in the driving process using advanced driving assistance system. Advanced driver assistance system consists of collision avoidance system, blind spot system and many more systems. This system is developed for safety and better driving. This system based upon vehicle to vehicle or vehicle to infrastructure system etc. In lane detection there are many approaches that are applied like feature based and model based. Feature based approach are used to detect edges and model based approach is a type of curve model. It supports various applications like lane departure warning, lane keeping assists, lane centring etc. In this paper we enhance the technique (additive Hough transform) using parallelism. Lane detection is an essential part in Lane Departure Warning System (LDWS). It is a protection system being able of warning the driver when the vehicle starts to move out of its lane and decrease traffic accidents efficiently. Lane Departure Warning System is also a most important part of Intelligent Vehicle technology. Intelligent vehicles are estimated to be capable to identify lane direction, sense objects and transmit to vehicular network for the avoidance of future accidents, or inform drivers of lane departure.

\section{A) Parallelism Approaches}

\section{- Data Parallelism}

Data parallelism is an application of parallelization of computing across multiple processors in parallel processing environment. Data parallelism centres on distributing the data across various parallel computing nodes. Data parallelism is accomplished when each processor works exactly the same task on various pieces of distributed data. Data parallelism is quite different model and is based on applying the same operation in parallel on different elements of dataset. The advantage of data parallelism is that it uses simpler model.

- Control Parallelism

Control parallelism is also known as function parallelism and task parallelism. It is a form of parallelism of computer code across multiple processors in parallel computing environments. Control parallelism centers on distributing tasks concretely executed by processes or threads across various processors. It is used in many parallel languages. These processes are communicated and synchronize with each other through massage passing or other mechanism.

\section{RELATED WORK}

Chung et al. (2015) [1] has discussed about driver assistant system that is dependent on various methods of image. In this detect the road lane markings a good camera is attached on the vehicle in front window. A modified approach can be planned to help increase your 
own the HT method in a computationally efficient manner. Therefore help us in order to show many spot of the vehicle with respect to the lane lines. The obtained image sequences are examined and refined by the proposed process, which immediately detects the lane lines. The experiment result shows the performance that the device performs properly for lane detection and lane prediction.

Tan et al. (2014) [2] has discussed about appropriate and effective lane detection, specially the curve lane detection, may be the assumption of Lane Departure Warning System (LDWS) and Forward Collision Warning System (FCWS). In that paper, a robust curve lane detection method based on Improved River Flow (IRF). RANSAC approach is planned to detect curve lane below difficult conditions. The lane markings are arranged in to a near vision field of straight line and a far vision field of curve line.

Filonenko et al. (2015) [3] had proposed a technique that is used to identify the lane markings by applying benefit of parallel processing. A ROI is limited by the existing speed of a vehicle. The segmentation is accomplished between lane markings. The desired process is split into 3 steps. The very first is lane markings detection on the basis of probability of color. The second reason is the implementation of range clustering evolution to establish the surface course. The third reason is the curve fitting was applied lane markings. The technique was applying on various dataset to show their effectiveness.

Jung et al. (2013) [4] has proposed an effective lane detection algorithm for lane departure detection; that algorithm is suited to reduced processing energy techniques like vehicle black boxes. First, we remove choose factors, which are help factors, to remove a hypotheses as two lines. In this task, Haar-like functions are utilized, and that allows people to make use of important pictures to eliminate computational redundancy. Next, our algorithm verifies the theory applying describing rules. These rules are on the basis of prediction on the assumption that the camera is mounted at the middle of the vehicle. Finally, in case a lane is detected, then the lane departure detection stage is performed.

Bounini et al. (2015) [5] has mentioned about Advanced Driving Assistant methods, intelligent and autonomous cars are encouraging alternatives to improve road security, traffic problems and people's comfort. Such programs need advanced computer perspective algorithms that need effective computers with high-speed control capabilities. Maintaining cars on the highway till their location sometimes stays a good problem specially when operating at large speed. The very first principle job is powerful effective navigation, which can be usually based on process vision to obtain RGB pictures of the road for more complex processing. The $2^{\text {nd }}$ job may be the vehicle's powerful control based on their, speed and direction. This paper presents an exact and successful road boundaries and painted lines' detection algorithm for intelligent and autonomous vehicle. It includes Hough
Transform to initialize the algorithm at every time required, and Canny edges' detector, least-square technique and Kalman filtration to reduce the flexible ROI, estimate the near future road boundaries' location and lines parameters.

Kang et al. (2014) [6] has dedicated to creation inexpensive car system. In that paper,propose a driver assistant system that is based on front camera. The planned process contains two parts. First portion is vehicle detection component that is based on adaptive improving applying transform (EMCT). Second part is the lane detection component applying on RANSAC. The results of process suggest that equally segments conduct robustly and so it could be a trusted intelligent vehicle system.

Chen et al. (2013) [7] has presented a novel algorithm for car lane boundary detection that is based on effective contours. The algorithm employs an adaptive GMM that will cope with quick improvements for detecting the vehicles used with a kalman filtration system to produce pixel level movement vectors. A novel effective curve power term on the basis of the accumulation of movement trajectory. The results are shown for movie from an actual road world to exhibit the effectiveness of planned algorithm, without the necessity for road lane marks.

Han et al. (2015) [8] has presented a method of lane detection predicated on vanishing point estimation. We first estimate the vanishing point with probabilistic voting utilizing the strength of line segment, and then detect the lanes through the use of flooding watershed algorithm.

Cho et al. (2014) [9] had discussed that to be able to increase the lane detection recognition via Hough transform, we increased the algorithm by collection multi-ROI to reduce error rates and unrecognized the main external anything as a lane. And we used superresolution reconstruction to improve the image.

Kang et al. (2014) [10] has discussed the Multi-lane detection algorithms that have already necessary for different vehicle applications for safety. In the existing technique, visible functions are basics hints for multilane detection. But, because of visible functions, temperature, situation and range of the region. On another hand, conventional geometric estimation-based techniques, may be not depending on visible functions, are incorrect to frequency or horizontal movement. In that paper, the proposed technique is a robust multi-lane detection algorithm on the basis of road scenarios.

Yang et al. (2012) [11] has presented a lane detection method. The type of the lane is created by the camera, helping to make the parallel lane lines in actual world. The lines on the single area of lane have exactly the same geometric function that can be utilized to create lane line geometric design for detection. In this paper, the different images of lane the model classifications performed at the same time. The combination of the modules can be used to over come the common problems of lane detection.

Baykal et al. (2014) [12] has discussed about A lane detection system based on one bit transform is presented in this paper. The utilization of lane detection systems as 
driver assist systems is increasing. The real-time operation requirement of lane detection systems is an important aspect. In this paper, a method that detects if the vehicle is outside the lane boundaries, by applying a simple one bit transform to the acquired image and counting the number of 1 valued pixels is proposed. This approach requires low computing power and is easy to implement.

Tan et al. (2015) [13] has discussed about lane detection is becoming of large interest in the region of intelligent vehicles and it offers the basic information which is often put on the further development of Driving Assistance System. In this paper, the proposed technique of a lane detection system that is based on iterative searching and Random Sample Consensus (RANSAC) curve fitting. Implementation Results show the effectiveness of our technique which detects lanes at a high appropriate rate and may be used in vehicles.

Deusch et al. (2012) [14] has discussed the Robust lane detection. This is the precondition for advanced driver assistance systems like lane departure caution. While detecting the vehicle's lane is enough for lane departure warning, or autonomous driving functions. In that factor, a method of lane detection is based on multiple objects on filter is presented. This technique provides for directly considering the dependencies between multiple lanes. The proposed lane detection algorithm is placed on difficult situations of a rural road.

Hunjae Yoo et al. (2013) [15] has discussed that the Lane detection is very essential in lots of advanced driver-assistance systems, gradient values between lanes and roads vary with light changes, which helps in changes the performances of the systems. The proposed method provides a new gray-level image which uses an adaptive canny edge detector, Hough transform, and curve model fitting method.

\section{Proposed Methodology}

Following are the various steps of lane detection. Each step converts an image into new form. Proposed algorithm takes following steps shown in figure 1.

Step 1: Fig 2 is showing the input image for Lane detection. The road image is given in which some objects (vehicles) are passing through. Image is showing quite extra information like sides of the roads, vehicles, trees at road sides, poles etc.

Step 2: Now apply fork operator and divide image into multiple threads. Fork operator allows scheduler to divide the road image into sub parts so that it can run in parallel fashion.

Step 3: Fig 3 has shown the gray scale output of the input image shown in fig 2. The overall goal of this step is to reduce the amount of data and preserve amount of information required to color the lanes.

Step 4: Now apply segmentation and image binarization will be done to locate the lanes in capture image and also smoothing of the results will be done by eliminating the unwanted objects. Fig 4 shows the segmented image.

Step 5: Now apply canny edge detector to detect lane edges. Fig 5 has shown the edge detected image for lane detection using canny edge detector operator.

Step 6: Now apply additive modified Hough transform and dynamic thresholding that is used to detect the lanes. Thresholding is used to increase the accuracy. Fig 6 shows the color metrics.

Step 7: Now color the lanes. fig 7 has shown the final colored images. The lane is colored by using green color.

Step 8: The join operator is responsible for joining the sub images into a single one to return back to its user.

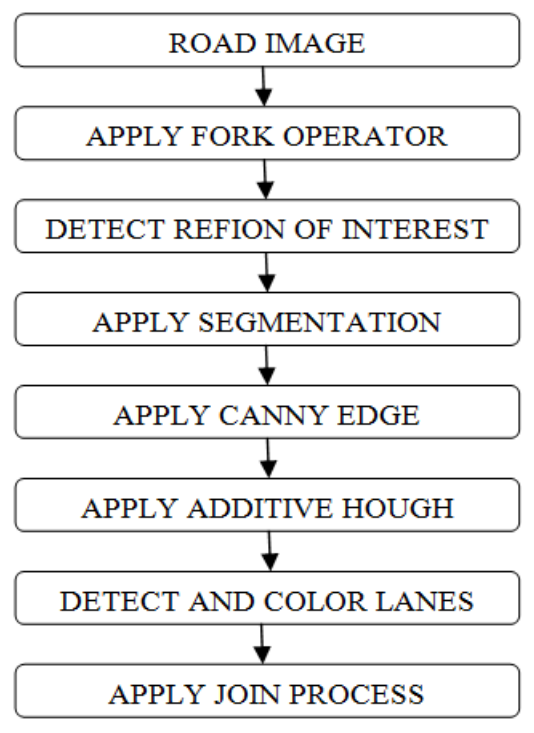

Fig.1. Proposed Method

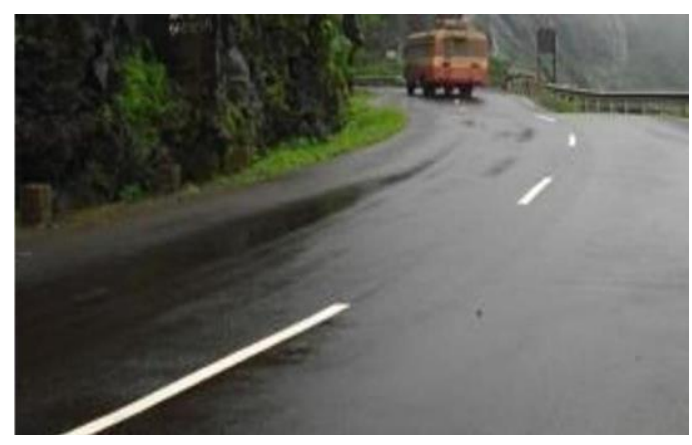

Fig.2. Input image

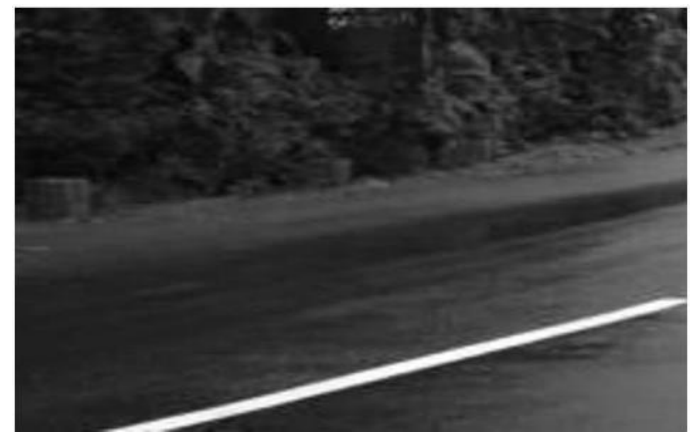

Fig.3. ROI 


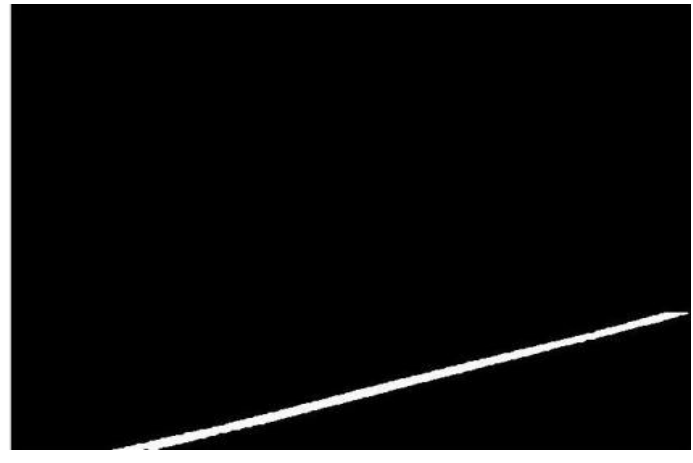

Fig.4. Segmented image

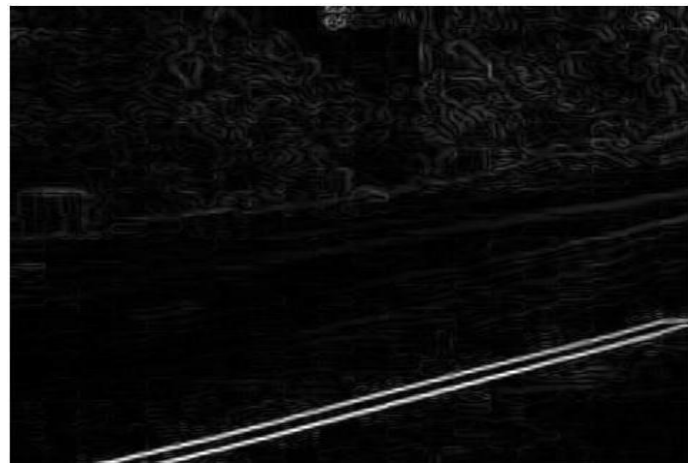

Fig.5. Canny image

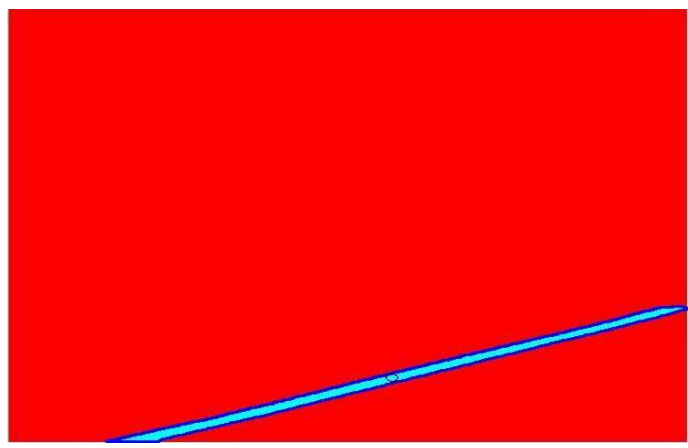

Fig.6. Color metric

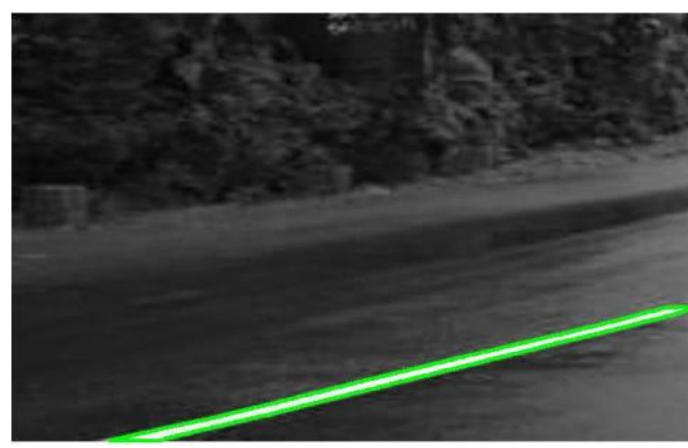

Fig.7. Output image

\section{RESULTS AND DISCISSIONS}

Following are the parameters that are used to improve the performance of proposed work. The comparison between the existing and proposed methods is shown by various parameters.

\section{A) Algorithm Results}

Following are the parameters that are used to improve the performance of proposed algorithm work. It contains the set of images which are used in lane detection algorithm on MATLAB with the help image processing toolbox.

\section{Accuracy:}

Accuracy identifies the closeness of a calculated value to a standard or known value or Accuracy is defined as, "The ability of a measurement to match the actual value of the quantity being measured". Accuracy is calculated by following formula:

$$
A C C=\frac{T P+T N}{P+N}
$$

Where

$\mathrm{TP}=$ True Positive

$\mathrm{TN}=$ True Negative

$\mathrm{P}=$ Positive Instances

$\mathrm{N}=$ Negative Instances

Table 1. Comparison of accuracy

\begin{tabular}{|c|c|c|}
\hline Images & $\begin{array}{c}\text { Existing } \\
\text { readings }\end{array}$ & $\begin{array}{c}\text { Proposed } \\
\text { readings }\end{array}$ \\
\hline $\mathbf{1}$ & 0.4862 & 0.3955 \\
\hline $\mathbf{2}$ & 0.3231 & 0.2975 \\
\hline $\mathbf{3}$ & 0.0457 & 0.0265 \\
\hline $\mathbf{4}$ & 0.3233 & 0.0613 \\
\hline $\mathbf{5}$ & 0.2480 & 0.2103 \\
\hline $\mathbf{6}$ & 0.3826 & 0.0889 \\
\hline $\mathbf{7}$ & 0.4960 & 0.4695 \\
\hline $\mathbf{8}$ & 0.0956 & 0.0577 \\
\hline $\mathbf{9}$ & 0.4483 & 0.3654 \\
\hline $\mathbf{1 0}$ & 0.2597 & 0.1596 \\
\hline $\mathbf{1 1}$ & 0.1820 & 0.0044 \\
\hline $\mathbf{1 2}$ & 0.4025 & 0.2394 \\
\hline $\mathbf{1 3}$ & 0.2715 & 0.2235 \\
\hline $\mathbf{1 4}$ & 0.4869 & 0.4839 \\
\hline $\mathbf{1 5}$ & 0.4923 & 0.3786 \\
\hline & & \\
\hline
\end{tabular}

Table 1 shows the comparison between existing and proposed readings of the parameters accuracy between fifteen images.

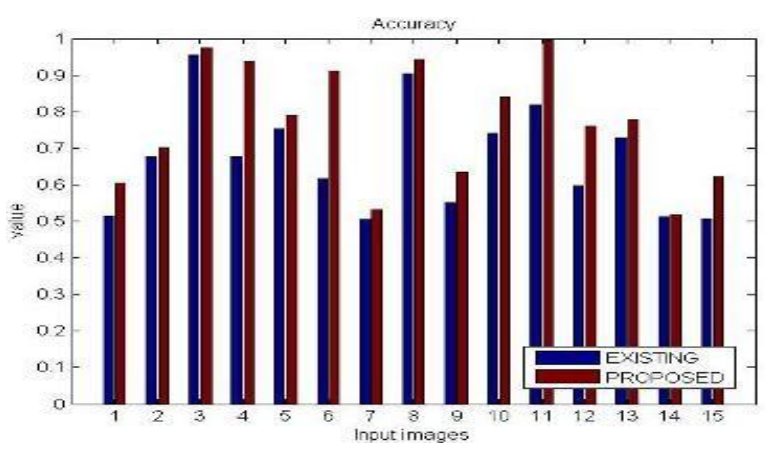

Fig.8. Bar graph of accuracy

The above graph proves that the results are improved in proposed technique. All the values of proposed work are as high as possible. 


\section{Error_Rate:}

The frequency with which errors or noise are presented to the channel. Error rate might be measured in terms of incorrect bits obtained per bits transmitted. Error rate is calculated by:

$$
\text { ERROR }_{\text {RATE }}=1-\text { Accuracy }
$$

Table 2. Comparison of error rate

\begin{tabular}{|c|c|c|}
\hline Images & $\begin{array}{c}\text { Existing } \\
\text { readings }\end{array}$ & $\begin{array}{c}\text { Proposed } \\
\text { readings }\end{array}$ \\
\hline $\mathbf{1}$ & 0.4862 & 0.3955 \\
\hline $\mathbf{2}$ & 0.3231 & 0.2975 \\
\hline $\mathbf{3}$ & 0.0457 & 0.0265 \\
\hline $\mathbf{4}$ & 0.3233 & 0.0613 \\
\hline $\mathbf{5}$ & 0.2480 & 0.2103 \\
\hline $\mathbf{6}$ & 0.3826 & 0.0889 \\
\hline $\mathbf{7}$ & 0.4960 & 0.4695 \\
\hline $\mathbf{8}$ & 0.0956 & 0.0577 \\
\hline $\mathbf{9}$ & 0.4483 & 0.3654 \\
\hline $\mathbf{1 0}$ & 0.2597 & 0.1596 \\
\hline $\mathbf{1 1}$ & 0.1820 & 0.0044 \\
\hline $\mathbf{1 2}$ & 0.4025 & 0.2394 \\
\hline $\mathbf{1 3}$ & 0.2715 & 0.2235 \\
\hline $\mathbf{1 4}$ & 0.4869 & 0.4839 \\
\hline $\mathbf{1 5}$ & 0.4923 & 0.3786 \\
\hline & & \\
\hline
\end{tabular}

The above table 2 shows the comparison between existing and proposed readings of the parameters accuracy between fifteen images.

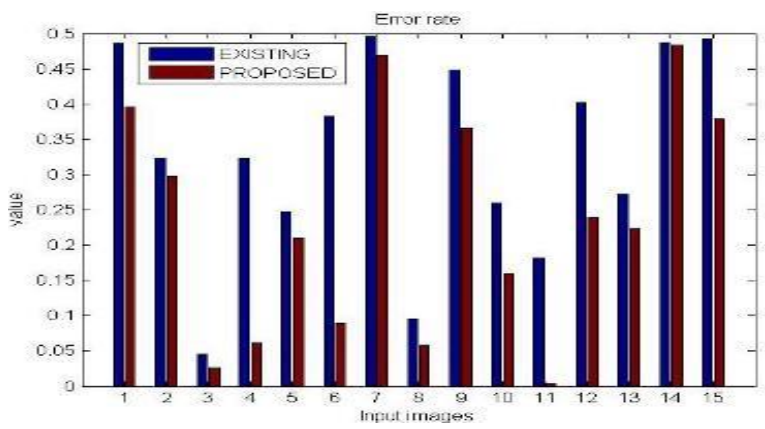

Fig.9. Bar graph of error rate

The above graph Fig 9 proves that the results are improved in proposed technique. All the values of proposed work are as low as possible.

\section{Execution_Time:}

It is described as the time taken to accomplish an application after submission to a device until finish. Execution time is found by tic-toc command. Place tic command is used before the first line of code and toc command is used after the last line of the code.

Table 3 shows the comparison between existing and proposed readings of the parameters accuracy between fifteen images. It can be observed from the table the values of proposed technique are low as comparative to existing technique.
Table 3. Comparison of execution time

\begin{tabular}{|c|c|c|}
\hline Images & $\begin{array}{c}\text { Existing } \\
\text { readings }\end{array}$ & $\begin{array}{c}\text { Proposed } \\
\text { readings }\end{array}$ \\
\hline $\mathbf{1}$ & 34.1775 & 16.6352 \\
\hline $\mathbf{2}$ & 52.8185 & 36.9117 \\
\hline $\mathbf{3}$ & 41.0394 & 24.7151 \\
\hline $\mathbf{4}$ & 32.2334 & 13.9466 \\
\hline $\mathbf{5}$ & 15.3657 & 13.7633 \\
\hline $\mathbf{6}$ & 10.5594 & 8.8614 \\
\hline $\mathbf{7}$ & 9.8691 & 9.3270 \\
\hline $\mathbf{8}$ & 34.4288 & 23.3369 \\
\hline $\mathbf{9}$ & 25.3326 & 13.1165 \\
\hline $\mathbf{1 0}$ & 15.3453 & 10.5365 \\
\hline $\mathbf{1 1}$ & 16.5909 & 12.6780 \\
\hline $\mathbf{1 2}$ & 28.4304 & 15.6047 \\
\hline $\mathbf{1 3}$ & 22.9703 & 14.2607 \\
\hline $\mathbf{1 4}$ & 11.9768 & 11.2388 \\
\hline $\mathbf{1 5}$ & 15.5275 & 15.4204 \\
\hline
\end{tabular}

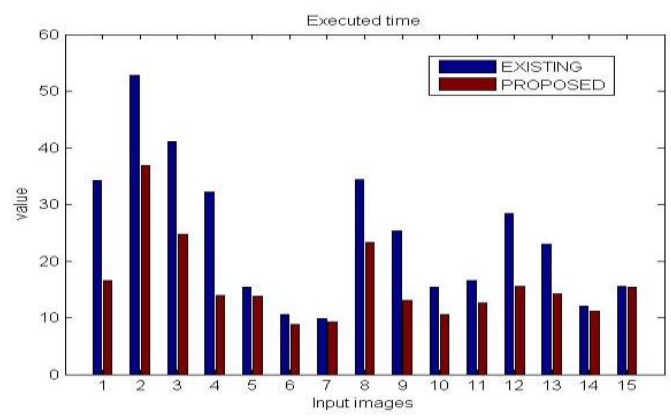

Fig.10. Bar graph of execution_time

The above graph proves that the results are improved in proposed technique. All the values of proposed work are as low as possible as compared to existing work.

\section{Overhead:}

It is defined as the extra time required by the system to attain the required goal. It is also called communication time.

Table 4. Comparison of overhead

\begin{tabular}{|c|c|c|}
\hline Images & $\begin{array}{c}\text { Existing } \\
\text { readings }\end{array}$ & $\begin{array}{c}\text { Proposed } \\
\text { readings }\end{array}$ \\
\hline $\mathbf{1}$ & 6.1671 & 2.6394 \\
\hline $\mathbf{2}$ & 13.6259 & 11.3184 \\
\hline $\mathbf{3}$ & 7.8858 & 7.5738 \\
\hline $\mathbf{4}$ & 6.2776 & 2.6376 \\
\hline $\mathbf{5}$ & 5.3370 & 2.3904 \\
\hline $\mathbf{6}$ & 5.0826 & 1.2313 \\
\hline $\mathbf{7}$ & 4.8162 & 0.9391 \\
\hline $\mathbf{8}$ & 7.1761 & 6.0580 \\
\hline $\mathbf{9}$ & 5.3759 & 2.4428 \\
\hline $\mathbf{1 0}$ & 5.1660 & 1.5872 \\
\hline $\mathbf{1 1}$ & 5.3447 & 3.2391 \\
\hline $\mathbf{1 2}$ & 5.6855 & 3.1482 \\
\hline $\mathbf{1 3}$ & 5.8640 & 2.5999 \\
\hline $\mathbf{1 4}$ & 4.7341 & 1.1243 \\
\hline $\mathbf{1 5}$ & 6.1040 & 1.6275 \\
\hline & &
\end{tabular}

Table 4 shows the comparison between existing and proposed readings of the parameters accuracy between fifteen images. It can be observed from the table the values of proposed technique are low as comparative to existing technique. 


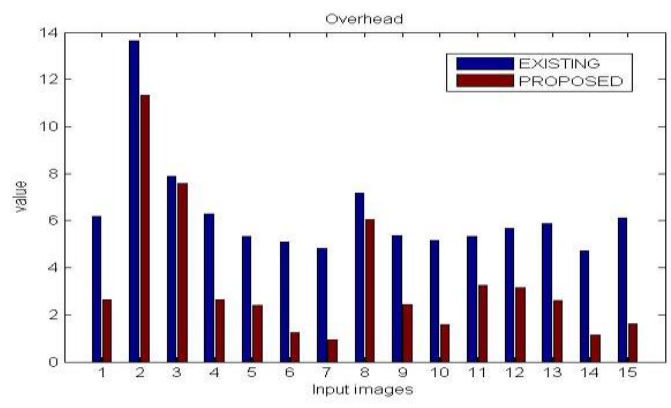

Fig.11. Bar graph of overhead

The above graph proves that the results are improved in proposed technique. All the values of proposed work are as low as possible as compared to existing work.

\section{B) Parallelism Results}

Following are the parameters that are used to improve the performance of proposed work. It contains the set of images which are used in lane detection algorithm on MATLAB with the help parallel processing toolbox.

\section{Speed_Up:}

It is the ratio of sequential execution time (when the algorithm is executed sequentially to the execution time) and parallel execution time (when the same algorithm is executed parallely to the execution time). Speed up can be mathematically represented as: $\mathrm{Sp}=\mathrm{Ts} / \mathrm{Tp}$, where Ts is the sequential execution time, $\mathrm{Tp}$ is the parallel execution time.

$$
S P E E D U P=\frac{\text { sequential execution time }}{\text { parallel execution time }}
$$

\section{Efficiency:}

It is a measure of processor utilization. Efficiency is a performance metrics closely related to speed up. It is the ratio of parallel execution time and the number of processors.

$$
\text { EFFICIENCY }=\frac{\text { parallel execution time }}{4}
$$

Table 5. Table shows speed and efficiency

\begin{tabular}{|c|c|c|}
\hline Images & Speed & Efficiency \\
\hline $\mathbf{1}$ & 4.1953 & 1.4916 \\
\hline $\mathbf{2}$ & 7.4076 & 1.1178 \\
\hline $\mathbf{3}$ & 6.0195 & 1.1431 \\
\hline $\mathbf{4}$ & 3.4964 & 1.0597 \\
\hline $\mathbf{5}$ & 3.5126 & 1.1426 \\
\hline $\mathbf{6}$ & 2.2718 & 1.1670 \\
\hline $\mathbf{7}$ & 2.0057 & 1.1426 \\
\hline $\mathbf{8}$ & 6.0183 & 1.0715 \\
\hline $\mathbf{9}$ & 3.5274 & 1.0113 \\
\hline $\mathbf{1 0}$ & 3.8355 & 1.3617 \\
\hline $\mathbf{1 1}$ & 3.3557 & 1.0664 \\
\hline $\mathbf{1 2}$ & 3.9110 & 1.0291 \\
\hline $\mathbf{1 3}$ & 3.6821 & 1.0475 \\
\hline $\mathbf{1 4}$ & 2.8259 & 1.0432 \\
\hline $\mathbf{1 5}$ & 3.3110 & 1.0905 \\
\hline
\end{tabular}

\section{Execution_Time:}

Execution time is described as the time taken performs an application after submission to a machine until finish. When the application is presented to a sequential computer, the execution time is known as sequential execution time and when application is presented to a parallel computer, the execution time is known as parallel execution time.

Table 6. Comparison of execution_time

\begin{tabular}{|c|c|c|}
\hline Images & $\begin{array}{c}\text { Parallel } \\
\text { proposed }\end{array}$ & $\begin{array}{c}\text { Sequential } \\
\text { proposed }\end{array}$ \\
\hline $\mathbf{1}$ & 16.7814 & 25.0322 \\
\hline $\mathbf{2}$ & 29.6307 & 33.1218 \\
\hline $\mathbf{3}$ & 24.0780 & 27.5254 \\
\hline $\mathbf{4}$ & 13.9857 & 14.8218 \\
\hline $\mathbf{5}$ & 14.0504 & 16.0548 \\
\hline $\mathbf{6}$ & 9.0873 & 10.6054 \\
\hline $\mathbf{7}$ & 8.0231 & 10.3251 \\
\hline $\mathbf{8}$ & 24.0735 & 25.7965 \\
\hline $\mathbf{9}$ & 14.1096 & 14.2694 \\
\hline $\mathbf{1 0}$ & 15.3421 & 20.8921 \\
\hline $\mathbf{1 1}$ & 13.4231 & 14.3152 \\
\hline $\mathbf{1 2}$ & 15.6442 & 16.1003 \\
\hline $\mathbf{1 3}$ & 14.7287 & 15.4285 \\
\hline $\mathbf{1 4}$ & 11.3036 & 11.7921 \\
\hline $\mathbf{1 5}$ & 13.2441 & 14.4437 \\
\hline
\end{tabular}

Table 6 shows the comparison between parallel proposed and sequential proposed readings of the parameters execution time between fifteen images. It can be observed from the table the values after converting parallel are low as comparative to sequential (means without parallel) technique.

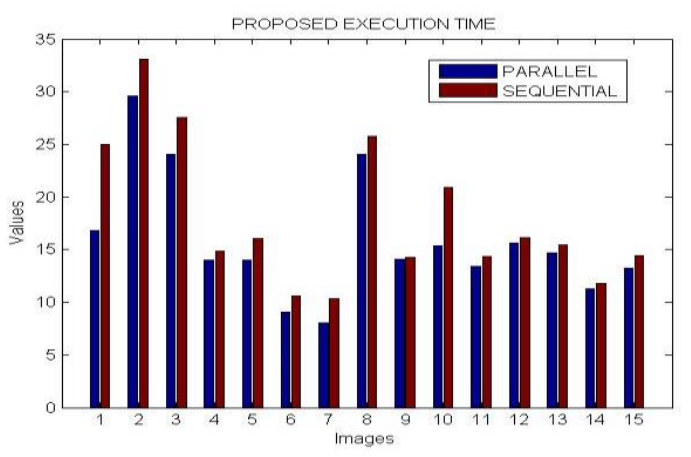

Fig.12. Bar graph of execution time

The above graph proves that the results are improved in proposed technique. All the values of proposed work are as low as possible as compared to existing work.

\section{CONCLUSION}

In this paper the implementation of the proposed algorithm is performed in MATLAB and convert the algorithm into parallelism by using parallel processing toolbox. Various road images have been taken and proposed algorithm is used on it. In this research work, the comparison between the existing and proposed methods are shown by various parameters The execution 
time for serial and parallel process is also compared in this work The comparison shows that proposed technique shows better results than the existing technique.

\section{REFERENCES}

[1] Yi, Shu-Chung, Yeong-Chin Chen, and Ching-Haur Chang. "A lane detection approach based on intelligent vision." Computers \& Electrical Engineering 42 ,23-29 (2015).

[2] Huachun Tan; Yang Zhou; Yong Zhu; Danya Yao; Keqiang $\mathrm{Li}$, "A novel curve lane detection based on Improved River Flow and RANSA," in Intelligent Transportation Systems (ITSC), 2014 IEEE 17th International Conference on, vol., no., pp.133-138, 8-11 Oct. 2014.

[3] A. Filonenko, D. C. Hernández, L. Kurnianggoro, D. Seo and K. H. Jo, "Real-time lane marking detection," Cybernetics (CYBCONF), 2015 IEEE 2nd International Conference on, Gdynia, 2015, pp. 125-128.

[4] Heechul Jung; Junggon Min; Junmo Kim, "An efficient lane detection algorithm for lane departure detection," in Intelligent Vehicles Symposium (IV), 2013 IEEE, vol., no., pp.976-981, 23-26 June 2013.

[5] F. Bounini, D. Gingras, V. Lapointe and H. Pollart, "Autonomous Vehicle and Real Time Road Lanes Detection and Tracking," Vehicle Power and Propulsion Conference (VPPC), 2015 IEEE, Montreal, QC, 2015, pp. $1-6$.

[6] J. S. Kang, J. Kim and M. Lee, "Advanced driver assistant system based on monocular camera," 2014 IEEE International Conference on Consumer Electronics (ICCE), Las Vegas, NV, 2014, pp. 55-56.

[7] Zezhi Chen; Ellis, T., "Automatic lane detection from vehicle motion trajectories," in Advanced Video and Signal Based Surveillance (AVSS), 2013 10th IEEE International Conference on, vol., no., pp.466-471, 27-30 Aug. 2013.

[8] Ju Han, Yoo Dong, Hwan Kim and Sung-Kee Park, "A new lane detection method based on vanishing point estimation with probabilistic voting," 2015 IEEE International Conference on Consumer Electronics (ICCE), Las Vegas, NV, 2015, pp. 204-205.

[9] Jae-Hyun Cho; Tsogtbaatar, E.; Seong-Hoon Kim; YoungMin Jang; Pham-Minh-Luan Nguyen; Sang-Bock Cho, "Improved lane detection system using Hough transform with super-resolution reconstruction algorithm and multiROI," in Electronics, Information and Communications (ICEIC), 2014 International Conference on, vol., no., pp.1-4, 15-18 Jan. 2014.

[10] Seung-Nam Kang; Soomok Lee; Junhwa Hur; Seung-Woo Seo, "Multi-lane detection based on accurate geometric lane estimation in highway scenarios," in Intelligent Vehicles Symposium Proceedings, 2014 IEEE, vol., no., pp.221-226, 8-11 June 2014.

[11] Jianyu Yang; Zhuo Li; Liangchao Li, "Lan detection based on classification of lane geometrical model," in Signal Processing (ICSP), 2012 IEEE 11th International Conference on , vol.2, no., pp.842-846, 21-25 Oct. 2012.

[12] Baykal, B.; Ozcan, A.R.; Erturk, S., "Lane detection system based on one bit transform," in Signal Processing and Communications Applications Conference (SIU), 2014 22nd, vol., no., pp.2237-2240, 23-25 April 2014.

[13] Tao Tan, Shouyi Yin, Peng Ouyang, Leibo Liu and Shaojun Wei, "Efficient lane detection system based on monocular camera," 2015 IEEE International Conference on Consumer Electronics (ICCE), Las Vegas, NV, 2015, pp. 202-203.

[14] Deusch, H.; Wiest, J.; Reuter, S.; Szczot, M.; Konrad, M.; Dietmayer, K., "A random finite set approach to multiple lane detection," in Intelligent Transportation Systems (ITSC), 2012 15th International IEEE Conference on, vol., no., pp.270-275, 16-19 Sept. 2012

[15] Hunjae Yoo; Ukil Yang; Kwanghoon Sohn, "GradientEnhancing Conversion for Illumination-Robust Lane Detection," in Intelligent Transportation Systems, IEEE Transactions on , vol.14, no.3, pp.1083-1094, Sept. 2013.

[16] Braga de Paula, M.; Rosito Jung, C., "Real-Time Detection and Classification of Road Lane Markings," in Graphics, Patterns and Images (SIBGRAPI), 2013 26th SIBGRAPI - Conference on, vol., no., pp.83-90, 5-8 Aug. 2013.

[17] Jun Wang; Tao Mei; Bin Kong; Hu Wei, "An approach of lane detection based on Inverse Perspective Mapping," in Intelligent Transportation Systems (ITSC), 2014 IEEE 17th International Conference on, vol., no., pp.35-38, 811 Oct. 2014.

[18] R. Gopalan, T. Hong, M. Shneier and R. Chellappa, "A Learning Approach Towards Detection and Tracking of Lane Markings," in IEEE Transactions on Intelligent Transportation Systems, vol. 13, no. 3, pp. 1088-1098, Sept. 2012.

[19] X. Li, Q. Wu, Y. Kou, L. Hou and H. Yang, "Lane detection based on spiking neural network and hough transform," 2015 8th International Congress on Image and Signal Processing (CISP), Shenyang, 2015, pp. 626-630.

[20] Chien Tsung-Yu; Chung Sheng-Luen, "Android-based driving assistant for lane detection and departure warning," in Control Conference (CCC), 2014 33rd Chinese, vol., no., pp.174-179,28-30July2014.

\section{Authors' Profiles}

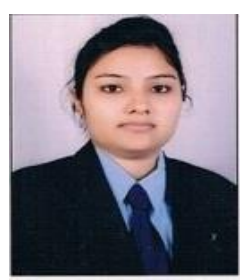

Amandeep Katru was born in Amritsar in 1992. She is a M.Tech student of the department computer engineering and technology at Guru Nanak Dev University. She obtained B.Tech degree from Computer science department from Amritsar College of enggineering and technology, Amritsar in 2014. She is currently working on her thesis on the topic lane detection.

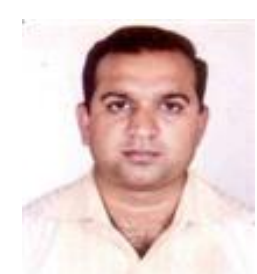

Anil Kumar is an Assistant Professor of the department Computer Engineering and Technology at Guru Nanak Dev University. He received his M.Tech degree from Punjabi University, Patiala in 2004 and B.Tech degree from Guru Nanak Dev University in 2000. He is currently Pursuing Ph.D from Guru Nanak Dev University, Amritsar. His area of interest in parallel computing. 
How to cite this paper: Amandeep Katru, Anil Kumar,"Improved Parallel Lane Detection Using Modified Additive Hough Transform", International Journal of Image, Graphics and Signal Processing(IJIGSP), Vol.8, No.11, pp.10-17, 2016.DOI: 10.5815/ijigsp.2016.11.02 